\title{
Technology connects patients to tertiary care for non-COVID illnesses in pandemic times: A case study from India
}

Jitender Kumar ${ }^{1}$, Tarundeep Singh ${ }^{2}$, Amarjeet Singh ${ }^{3}$

${ }^{1}$ Junior Resident, Department of Community Medicine and School of Public Health, Postgraduate Institute of Medical Education and Research, Chandigarh; ${ }^{2}$ Associate Professor, Department of Community Medicine and School of Public Health, Postgraduate Institute of Medical Education and Research, Chandigarh; ${ }^{3}$ Professor, Department of Community Medicine and School of Public Health, Postgraduate Institute of Medical Education and Research, Chandigarh.

\begin{tabular}{|c|c|c|c|c|c|c|c|}
\hline Abstract & Introduction & Methodology & Results & Conclusion & References & Citation & Tables / Figures \\
\hline
\end{tabular}

\section{Corresponding Author}

Dr Tarundeep Singh, Associate Professor, Department of Community Medicine and School of Public Health, Postgraduate Institute of Medical Education and Research, 160012, Chandigarh

E Mail ID: tarundeep.singh@gmail.com

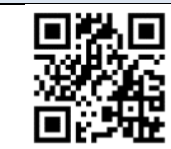

\section{Citation}

Kumar J, Singh T, Singh A. Technology connects patients to tertiary care for non-COVID illnesses in pandemic times: A case study from India. Indian J Comm Health. 2021;33(1):202-204. https://doi.org/10.47203/IJCH.2021.v33i01.029

Source of Funding: Nil Conflict of Interest: None declared

\section{Article Cycle}

Received: 28/12/2020; Revision: 15/01/2021; Accepted:03/02/2021; Published:31/03/2021

This work is licensed under a Creative Commons Attribution 4.0 International License.

\section{Abstract}

During the COVID-19 pandemic times, non-COVID patients faced many difficulties to access health care because of diversion of health resources to manage the pandemic, partial or complete closure of routine OPD services and travel restrictions due to lockdown. Increased use of technology enhanced the effectiveness of the healthcare system to maintain treatment avenues in this challenging time. Also, the pandemic accelerated diffusion of technological innovations like teleconsultation among healthcare workers and patients. The present case study is about a young, breast cancer, female patient from rural India who was diagnosed with the disease just before the pandemic times. She was put on neo-adjuvent chemotherapy in a tertiary care hospital about 150 kms away from her native place but meanwhile lockdown was imposed in India to control COVID-19 spread. She was maintained on follow up through teleconsultation services. She was facilitated through travel restrictions for chemotherapy cycles by e-pass. Her concerns regarding disease, treatment options, follow ups, effect of treatment on her fertility and adverse effects of chemotherapy during her treatment course were satisfactorily addressed through teleconsultation. Necessary investigations done nearby were sent digitally to treating doctor that minimized her visits. After completion of chemotherapy she underwent breast conservation surgery.

\section{Keywords}

Telemedicine; Technology; COVID-19; Lockdown

\section{Introduction}

Corona Virus Disease (COVID-19) itself, and its control measures have disrupted health care systems worldwide. Resources in healthcare have been diverted towards combating COVID-19, compromising routine healthcare. To minimize impact of COVID-19, India observed nationwide

graded strict lockdowns (Phases 1-6) from March 24, 2020 followed by partial restrictions. Travel got restricted and patients with non-COVID-19 illnesses faced difficulty in getting timely care. During this challenging time, information technology came to the rescue of the patients to access treatment and get basic information about their malady. 
This case study highlights the role of technology in enhancing outreach of healthcare system to maintain treatment avenues for non-COVID illnesses in pandemic times in a developing country.

Case: A 27 years old healthy and educated lady, a resident of small town about $150 \mathrm{Kms}$ away from a tertiary healthcare institution noticed a lump in her breast in January, 2020. Patient had no family history of breast cancer in first degree relatives and was not exposed to any modifiable risk factors for the disease. She believed that she was too young to develop breast cancer. Few of her acquaintances, who had breast cancer, were in their old age. She was neither aware of breast self-examination and other screening methods for breast cancer nor she ever underwent such procedure. Even after she noticed swelling in her breast, she was hesitant to consult a nearby male surgeon and was willing to travel about $60 \mathrm{~km}$ to get herself examined by a female doctor. It was only after her husband's motivation that she consulted the nearby surgeon who facilitated her diagnosis.

During the lockdown, she contacted the tertiary care institution through tele-consultation. She was called for biopsy. She applied online for an e-pass to travel amidst travel restrictions. The biopsy showed infiltrating duct carcinoma. She was scheduled for neo-adjuvant chemotherapy, a total of 8 cycles with AC-T regimen. Considering her young age and her wish to conceive in future, she was started on $\mathrm{GnRH}$ analogue in order to preserve her ovarian functions. Travel across the states was restricted and allowed only with e-pass. She used to apply for COVID-19 epass before her scheduled visit to nearest tertiary care center for chemotherapy. Her concerns regarding treatment were addressed through teleconsultation for which contact number of treating doctors was provided to her. She was asked to contact in case of any query or issue. She contacted the doctors a couple of times. Her queries were addressed quickly and satisfactorily.

According to the patient, this service was helpful during her treatment course as she could contact her doctor and share investigation reports whenever she was in need without travelling distances. She was asked to get herself admitted after COVID-19 negative report for surgical intervention.

Patient was worried if only the diseased part would be removed or whole breast. She could not discuss this with her treating doctor. After her diagnosis, the patient read about the disease on internet. She posed her queries to treating doctors during her visits for chemotherapy.

Patient Perspective: To gauge her perception how different her life will be if her entire left breast is removed during surgery she replied "since when I came to know about my disease, I read about it from internet sources. I made my mind from starting that I will lose my breast. Purpose of the treatment is to get rid of the disease". Will her relationships with her husband change? She replied, "I have discussed with my husband regarding this. He has always shown his support to me. He said he has no issues with that". How will her social perception change? "I will have full confidence while in public because I have not invited this disease. It can occur to anyone", she replied.

Patient is in child bearing age and has a two years old child. She was asked about her perceptions regarding future pregnancies. About her plan regarding her family size she replied, "I had planned for having two children. I wish to have one more child". She thinks if she can get pregnant in future? "I was told by my doctor that chances of getting pregnant after chemotherapy will be quite low, But, if I complete course of $\mathrm{GnRH}$ analogue injections, chances will increase substantially. I have no idea if I will be able to conceive or not. But I will conceive only after consultation with doctors. If they assure me that my medications will not affect my baby adversely, I will plan for next pregnancy".

Concerning the risk of breast cancer to female child if she has any in future, she replied, "She can also have the disease. But then why I had this disease when I had no family history? It is all about chance. Nothing is certain". Patient was also aware about recurrence and need for follow ups. She said, "My doctor has explained to me about recurrence of this disease. This disease can reoccur in same breast or in the other breast.

Patient was found medically fit for surgery. Eventually, she had lumpectomy and she was happy that her breast got preserved.

\section{Discussion}

Indian Space Research Organization (ISRO) initiated telemedicine in India as a pilot in 2001 with the hope that telemedicine will decrease the burden of hospitals, need of transport and save resources of people.(1) Despite great potential, telemedicine could not have the effect it could have. Barriers like lack of formal organizational structure, lack of 
standard operating procedures for tele-consultation practice and low internet connectivity impeded its development. Reluctance of both physicians and patients to use information technology was due to difficulty in working with modern gadgets, fear of medical indemnity and resistance to change. Physicians believe that patient consultation and treatment are incomplete without touching the patient. (2)

Innovation diffusion theory by Roger talks about five significant characteristics of an innovation which decide whether that innovation will be adopted by users or not. These are: relative advantage, compatibility, complexity, trialability and observability. An innovation is more likely to be adopted if it has a relative advantage over the existing method, it is compatible with existing values and needs, it is easy to understand and use, it can be experimented and the results of its use are observable. (3) COVID-19 has helped in diffusion of the telemedicine innovation among its users that includes both healthcare providers and patients. During the lockdown phase after 25th March, the Government of India issued the Telemedicine Practice Guidelines and legitimized the use of teleconsultation by registered medical practitioners. (4) The lockdown has increased the usage of internet and communication technology to access the health care system in wake of restrictions brought in due to the pandemic and resultant control measures. At the same time reluctance or apathy of healthcare providers to use tele-consultation decreased and development of easy to use platforms or mechanisms for appointment, consultation and prescription, many as startups facilitated teleconsultation process.(5) Widespread use of smartphones with high resolution camera and high speed internet help to share images between doctors and patients and growing interest in noise cancelling headphones or microphones to aid in better quality of consultations.

This case highlights the role of internet and information technology to overcome barriers to treatment faced by a breast cancer patient during lockdown imposed in India due to COVID-19. The potential delays were very aptly prevented by use of Tele-consultation besides addressing concerns and maintaining proper follow up. It also sought to educate patients in self-care by virtually connecting them to their treating doctors, share their reports digitally and schedule their visits to hospitals online. However, use of this technology remains limited by access to technology, awareness, education status and technology friendliness of the user needs to be encouraged.

\section{References}

1. Mishra SK, Kapoor L, Singh IP. Telemedicine in India: current scenario and the future. Telemed J E Health. 2009;15(6):568-75. doi: 10.1089/tmj.2009.0059. PMID: 19659413.[PubMed].

2. Sakakibara R, Doi H, Fukudo S. Lewy body constipation. J Anus Rectum Colon. 2019;3(1):10-17. doi: 10.23922/jarc.2018-022. PMID: 31559362; PMCID: PMC6752132.[PubMed]

3. Rogers E. Diffusion of Innovations. 4th edn. New York: Free Press, 1995

4. Telemedicine Practice Guidelines - Enabling Registered Medical Practitioners to Provide Healthcare Using Telemedicine. Appendix 5 of the Indian Medical Council (Professional Conduct, Etiquette and Ethics Regulation) 2002. Available from: https://www.mohfw.gov.in/pdf/Telemedicine.pdf [accessed on 26 Feb 2021].

5. Singh I, Chand K, Singh A, Kandadi KR. Time for a culture change: understanding and reducing risk, morbidity and mortality from COVID-19 in those of black and minority ethnicity. Br J Hosp Med (Lond). 2020;81(5):1-4. doi: 10.12968/hmed.2020.0241. Epub 2020 May 22. PMID: 32468944.[PubMed]. 